\title{
EUVE PHOTOMETRIC ECLIPSE OBSERVATIONS OF AR LACERTAE
}

\author{
D. J. Christian, J. J. Drake, ${ }^{1}$ R. J. Patterer, ${ }^{2}$ P. W. Vedder, AND S. Bowyer \\ Center for EUV Astrophysics, 2150 Kittredge Street, University of California, Berkeley, California 94720 \\ Electronic mail: \\ Received 1994 April 21; revised 1996 May 13
}

\begin{abstract}
We report on observations of the eclipsing RS CVn system AR Lac with the Extreme Ultraviolet Explorer (EUVE) scanning and deep survey (DS) telescopes. This system consists of a G2 IV star and a K0 IV star separated by $9.2 R_{\odot}$ with an orbital period of about 1.98 days. The scanner observations consisted of a single pointing over a period of 25 hours, providing some coverage of both primary and secondary eclipses, and subsequent scanning during the all-sky survey. During the pointed observation AR Lac was detected in the EUVE Lexan/boron (Lexan/B, 50-180 $\AA$ ) and Al/Ti/C (160-240 $\AA$ ) bands at average count rates of 0.12 and 0.016 count s $^{-1}$, respectively. During primary eclipse, the Lexan/B count rate was at about one-third of its subsequent value at quadrature, suggesting that, in the case of a compact and relatively homogeneous corona, a minimum of $60 \%-70 \%$ of the extreme ultraviolet (EUV) emission originates on the G star. There was no detectable modulation corresponding to secondary eclipse in either Lexan/B or Al/Ti/C bands. Data taken several months later during the $E U V E$ all-sky survey yield the same Lexan/B count rate as the pointed data but only an upper limit in the Al/Ti/C bandpass. AR Lac was observed again with the DS as part of a Guest Observer pointed observation. This single pointing lasted slightly more than three days and included both primary and secondary eclipses. AR Lac was detected in the DS Lexan/B band with an average count rate of 0.31 count s$^{-1}$. The primary eclipse is easily visible as a $\sim 40 \%$ decrease in intensity. A dip in the light curve is present at the time of secondary eclipse, but is not readily discernible from the average level of variability. A flare corresponding to a factor of 2 increase over the quiescent emission count rate was observed during the next expected secondary eclipse. We have performed an elementary emission measure analysis and compared isothermal models that fit the EUVE data to earlier Einstein, EXOSAT, ASCA, and ROSAT two-temperature models culled from the literature. In general, the EUVE data indicate very similar emission measures to those derived in previous work, indicating that the corona of AR Lac does not vary in average $\mathrm{x}$-ray or EUV luminosity over periods of years up to a decade. Assuming an isothermal model with $T \approx 10^{7} \mathrm{~K}$, as indicated by the emission measure analysis, the total Lexan/B count rate at quadrature implies an EUV luminosity of $1.1 \times 10^{30} \mathrm{erg} \mathrm{s}^{-1}$ in the 50-180 $\AA$ bandpass. (C) 1996 American Astronomical Society.
\end{abstract}

\section{INTRODUCTION}

RS CVn systems are binaries with orbital periods typically between 1 and 14 days. The strictest definition of the class (Hall 1976) requires at least one evolved component; systems in which both members are still on the main sequence are usually classified as BY Dra variables. Individual members have spectral types from late-F through early-M with the majority being G- or K-type stars. Because the orbital separation is small, tidal viscosity tends to synchronize the stellar rotation and orbital periods, increasing the rotation rates beyond typical values for single-late-type stars. This rapid rotation makes them strongly magnetically active and, therefore, strong sources of chromospheric and coronal emission. Indeed, in following with the rotation-activity paradigm, they are among the very brightest stellar coronal sources observed at short wavelengths. An insightful discus-

${ }^{1}$ Harvard-Smithsonian Center for Astrophysics, 60 Garden Street, Cambridge, MA 02138.

${ }^{2}$ Currently at Hughes STX, Goddard Space Flight Center. sion of the class can be found in Montesinos et al. (1988) and a comprehensive catalog can be found in Strassmeier et al. (1993).

AR Lac is the brightest known totally eclipsing RS CVn binary ${ }^{3}$ and is, therefore, especially interesting. The system is at a distance of $47 \mathrm{pc}$ (Strassmeier et al. 1993), has a period of 1.983 days and is composed of a G2 IV and a K0 IV star of approximately equal masses but unequal radii (Chambliss 1976; Popper \& Ulrich 1977), and separated by a distance of about $9.2 R_{\odot}$. We have adopted the system parameters of Chambliss (1976) that are summarized in Table 1. Because the G star is completely eclipsed and the orbital period is relatively short, this system has been a popular target for observers at all wavelengths. In the study of stellar coronae, the eclipses of systems like AR Lac are especially important because they can yield otherwise inaccessible information on the spatial geometry of coronal emission.

\footnotetext{
${ }^{3}$ Although $\epsilon \mathrm{UMi}$ is a brighter, totally eclipsing chromospherically active binary, AR Lac is the brightest RS CVn by the strict definition of Hall (1976).
} 
TABLE 1. AR Lac system parameters from Chambliss (1976).

\begin{tabular}{lcc}
\hline & Primary & Secondary \\
\hline Sp Type & G2 IV & K0 IV \\
Radius & $1.54 R_{\odot}$ & $2.81 R_{\odot}$ \\
Mass & $1.35 \mathscr{C l}_{\odot}$ & $1.35 \mathscr{M l}_{\odot}$ \\
Separation & & $9.22 R_{\odot}$ \\
Period & & 1.983 days \\
\hline \hline
\end{tabular}

The first clues as to the spatial structure of the AR Lac coronae came from radio observations: Owen \& Spangler (1977) failed to detect an eclipse in the quiescent radio emission, a result which suggested that the radio flux originates from a region that is large compared to the radii of the component stars. At higher energy, early Einstein Solid State Spectrometer (SSS) observations by Swank \& White (1980) yielded little spatial information because of insufficient temporal coverage. However, based on these data and observations of six other RS CVn systems, Swank et al. (1981) were able to distinguish apparently two distinct temperature components, centered on $\sim 7 \times 10^{6} \mathrm{~K}$ and $\leqslant 4 \times 10^{7} \mathrm{~K}$, in the coronae of these systems. They reported two different states of AR Lac's corona: one with temperatures $T_{1}=6.7 \times 10^{6} \mathrm{~K}$ and $T_{2}=9.3 \times 10^{7} \mathrm{~K}$; and the other with $T_{1}=7.4 \times 10^{6} \mathrm{~K}$ and $T_{2}=4.3 \times 10^{7} \mathrm{~K}$. The authors noted that, for their data, the low temperature component was virtually indistinguishable in the two states.

Subsequently, Walter et al. (1983) used the Imaging Proportional Counter (IPC) on Einstein to observe about $17 \%$ of the orbit, concentrating on the eclipses and quadratures. Based on these observations, they concluded that about $60 \%$ of the x-ray emission arises in a region around the $\mathrm{K}$ star with the remaining $40 \%$ attributable to the $G$ star. They deduced that the G-star corona could be approximated by a single isothermal component $\left(\sim 7 \times 10^{6} \mathrm{~K}\right)$ composed of a large number $\left(10^{5}-10^{6}\right)$ of magnetic loops with scale heights of about $0.02 R_{\star}$. Similarly, the $\mathrm{K}$ star could be modeled by two components: an inner component with relatively small scale height similar to that on the $\mathrm{G}$ star and an extended hot corona $\left(\sim 10^{7} \mathrm{~K}\right)$ with a scale height comparable to the stellar radius and confined to equatorial latitudes.

Substantial progress, though with partially conflicting results, has been made with the EXOSAT, ROSAT, and ASCA observatories. The high elliptical orbit of EXOSAT enabled continuous observations of a given target for up to three days. Observations of the eclipsing system Algol and of the partially eclipsing RS CVn, ER Vul, showed no strong x-ray modulation or eclipses, again suggesting a scale height for their coronae of several stellar radii (White et al. 1986; White et al. 1987). A detailed study of an EXOSAT observation covering one full orbit of AR Lac by White et al. (1990) produced results in qualitative agreement with those of Walter et al. (1983). No sign of orbital modulation was present in the medium energy proportional counter (ME), but strong orbital modulation was seen by the low energy imaging telescope (LEIT). This interesting result suggested that the two distinct temperature components proposed by Swank et al. (1981) were associated with physically different regions in the corona: the hot component being extended, with a height of several stellar radii; and the cooler component being much more compact, with a height smaller than the stellar radius. White et al. (1990) also used the EXOSAT data to make detailed maximum entropy models of the AR Lac coronae, yielding spatial information on the lower temperature corona. Their favored solutions suggested covering fractions of $\sim 16 \%$ and $\sim 25 \%$ on the $\mathrm{K}$ star and $\mathrm{G}$ star, respectively.

One interesting aspect of these models is the presence of a very large active region on the $\mathrm{G}$ star which contributes most of the G-star flux. Neff et al. (1989) found evidence for a large region on the G star in their IUE data (taken about one year after the EXOSAT data) which was chromospherically inactive in the $\mathrm{Mg}$ II lines at a similar surface location. It is unclear if the same region can be connected with both results. If so, it would be most puzzling to find an area of chromospheric inactivity associated with a very active region in the corona. Neff et al. (1989) also found three distinct bright regions on the $\mathrm{K}$ star and it is worth noting that the maximum entropy models of White et al. (1990) suggest the $\mathrm{K}$-star flux comes from all latitudes, including regions near the poles. Siarkowski (1992) has also modeled the EXOSAT data using a three-dimensional $\chi^{2}$ minimization technique. A notable feature arising from the modeling was a large, extended emission region between the two stars, apparently associated with the $\mathrm{K}$ star.

Recently Ottmann et al. (1993) have presented a detailed analysis of ROSAT PSPC observations of AR Lac obtained in 1990 June. These observations covered one orbital cycle. In contrast to the EXOSAT ME data presented by White et al. (1990), the ROSAT data show a clear primary eclipse at all energies, arguing against the spatially extended hot coronae proposed by White and coworkers. However, detailed modeling of the x-ray light curves did suggest the presence of prominent coronal features on each star, qualitatively similar to those suggested by White et al. (1990), but showed no evidence for extended emission over spatial scales similar to that of the binary separation.

ASCA observations obtained in 1993 June and analyzed by White et al. (1994) painted a similar picture. A $50 \%$ reduction in flux across the ASCA energy band (0.4-7 keV) was detected during primary eclipse, with a shallow minimum visible during secondary eclipse. Interestingly, a twotemperature plasma model fit to the data suggested metal abundances lower than solar by factors of 2-4. A follow-up analysis by Singh et al. (1996) produced similar results.

The existing EXOSAT, ROSAT, and ASCA observations of AR Lac cover a period of near 10 years: 1984 (EXOSAT); 1990 (ROSAT); and 1993 (ASCA). Since the optical patterns thought to be associated with dark surface spots analogous to sunspots exhibited by RS CVn stars (Hall \& Henry 1994) are known to evolve over periods of several months to years (the "photometric wave"), including that of AR Lac (e.g., Hall et al. 1976), it is most likely that the overall pattern of coronal activity (on top of stochastic variations) will also change over similar time scales. In this context, further contemporary observations of the coronal emission on AR Lac would be most interesting.

In this paper, we present recent observations of AR Lac 
performed with the EUVE scanning and Deep Survey (photometric) telescopes during the initial in-orbit calibration (IOC), all-sky survey, and Guest Observer (GO) phases of the mission. A preliminary analysis of the pointed scanner data were presented by Patterer et al. (1993). Here, we have rederived the count rates, fluxes, and light curves using new, up-to-date calibrations and software. We compare these data with light curves derived from the later Guest Observer (GO) observation (an independent analysis of these data has very recently been presented by Walter 1996), and we describe an emission measure analysis based on count rates observed in the EUVE $\mathrm{Al} / \mathrm{Ti} / \mathrm{C}$ and Lexan/B bandpasses. The results are discussed briefly in the light of previous work.

\section{OBSERVATIONS}

\subsection{Instrumentation}

$E U V E$ observed AR Lac during the 45 day IOC phase undertaken soon after launch and, subsequently, during the all-sky survey. It was used as a boresight calibration target for the three co-aligned scanning telescopes, referred to locally as Scanners A, B, and C. During the Guest Observer (GO) phase of the mission, it was also observed using the deep survey and spectrometer telescope (DSS).

Scanners A and B and the DSS telescope use WolterSchwarzschild Type I mirrors of gold-plated aluminum, while Scanner C is a Wolter-Schwarzschild Type II mirror of nickel-plated aluminum. All use $50 \mathrm{~mm} \mathrm{Z}$ stack microchannel plates as detectors: the detectors on Scanners A and B have a $\mathrm{MgF}_{2}$ photocathode, while Scanner $\mathrm{C}$ is bare. Each detector sits behind a filter holder that contains two different filters. Scanners A and B both have Lexan/B and Al/Ti/C filters, while Scanner $\mathrm{C}$ has a $\mathrm{Sn} / \mathrm{SiO}$ and $\mathrm{Ti} / \mathrm{Sb} / \mathrm{Al}$ filter, each filter covering half of the detector area. The filters are aligned so that an object being observed in Scanner A (Lexan/B) is simultaneously observed in the Scanner B (Al/ $\mathrm{Ti} / \mathrm{C})$ and Scanner $\mathrm{C}(\mathrm{Sn} / \mathrm{SiO})$. Correspondingly, an object in Scanner A $(\mathrm{Al} / \mathrm{Ti} / \mathrm{C})$ is simultaneously observed by Scanner B (Lexan/B) and Scanner C (Ti/Sb/Al). The filter bandpasses are: $50-180 \AA$ (Lex/B), 160-240 $\AA$ (Al/Ti/C), 345-605 (Ti/Sb/Al), and 500-740 $\AA$ ( $\mathrm{Sn} / \mathrm{SiO})$.

Photons gathered by the DSS telescope that are not intercepted by the three symmetrically oriented grazing incidence diffraction gratings which comprise the dispersive elements of the spectrometer fall on the Deep Survey (DS) detector. On-boresight photons, such as would be observed from a point source during a normal spectroscopic pointing, impinge on a Lexan/B filter similar to that of Scanners A and B (a boron coated Lexan substrate supported on a nickel mesh), which has significant transmission between about 65 and 190 $\AA$, peaking at about $90 \AA$ with an effective area of about 28 $\mathrm{cm}^{2}$.

A complete description of the EUVE instrument and its performance can be found in Welsh et al. (1989) and Bowyer \& Malina (1989).

\subsection{Calibration Pointing}

AR Lac was observed during the IOC phase from 1:26 UT on 1992 June 27 until 2:45 UT on 1992 June 28, a period that included 17 satellite orbits. Unfortunately, because of high geocoronal emission in the EUVE bandpasses during satellite daytime, only the night portion of each orbit is able to be used. This limits the exposure to about 20 minutes out of each 96 minute orbit. In addition, three of the night orbits were unusable because AR Lac was positioned near a pinhole in the Scanner A Lexan/B filter which is transparent to ultraviolet (UV) as well as extreme ultraviolet (EUV) and $\mathrm{x}$-ray radiation. Rather than trying to disentangle the UV and EUV radiation, we chose not to process the data from these three orbits. AR Lac was detected in the Lexan/B and Al/ $\mathrm{Ti} / \mathrm{C}$ filters but was not detected in the two longer wavelength filters. The lack of a detection in these last two filters is because of a combination of the steep increase in attenuation by the interstellar medium towards longer wavelengths and the decrease in the number of emission lines at longer wavelengths from the hot, $\sim 10^{7} \mathrm{~K}$, plasma which appears to characterize AR Lac's corona.

The raw EUVE telemetry was reduced using the End-toEnd System (EES) software described by Marshall (1989). The EES produced a "pigeonhole" file containing all photons received during valid time intervals within $0.2^{\circ}$ of the known source position. The pigeonhole data were then binned into full night orbits, typically $1200 \mathrm{~s}$. A circle of radius $0.08^{\circ}$ about the source center was used to determine the source counts. An annulus of inner radius $0.08^{\circ}$ and outer radius $0.18^{\circ}$ was used to estimate the number of background counts. The radius of the source circle was chosen to be equal to the $99 \%$ encircled energy radius determined from the telescope point-spread functions (PSFs). The net count rate was then corrected for electronic effects, vignetting, and the small sensitivity differences known to exist between one Scanner A Lexan/B quadrant and the other three Lexan/B filter quadrants from both scanners. The resulting count rates are listed in Table 2. For deriving these count rates, we have separated the periods when the star was in and out of primary eclipse (see Sec. 3).

The preliminary analysis of some of the IOC data presented by Patterer et al. (1993) has been improved upon in several respects. Both the data reduction procedures and instrument calibrations have been refined. Electronic deadtime effects have now been corrected for, and we have used much improved PSFs in determining the count rates. The average Lexan/B count rate has increased by about $40 \%$, a larger effect than was anticipated in our previous work. The average $\mathrm{Al} / \mathrm{Ti} / \mathrm{C}$ count rate has been revised upwards by about a factor of 3, with the bulk of the increase again coming from the improved PSFs and improved understanding of the electronic effects.

\subsection{All-Sky Survey}

AR Lac was observed again by EUVE during the all-sky survey. In survey mode the spacecraft was spun so that the scanning telescopes scanned along lines of ecliptic longitude. Typically the spacecraft would complete one scan during each nighttime orbit. Typical exposure times for a given object were $20 \mathrm{~s}$ per scan. The period of time for which an object remained along the scanning field of view is a function of ecliptic latitude. Objects at the ecliptic equator spent 
TABLE 2. Count rates and luminosities observed in the Lexan/B and $\mathrm{Al} / \mathrm{Ti} / \mathrm{C}$ bands (refer to text).

\begin{tabular}{|c|c|c|c|c|}
\hline & $\begin{array}{l}\text { Scanner Lexan/B } \\
\text { Count rate } \\
\left(\text { count s }{ }^{-1}\right)\end{array}$ & $\begin{array}{c}L_{30}{ }^{\mathrm{a}} \\
\left(\mathrm{erg} \mathrm{s}^{-1}\right)\end{array}$ & $\begin{array}{l}\text { Scaner } \mathrm{Al} / \mathrm{Ti} / \mathrm{C} \\
\text { Count rate } \\
\left(\text { count } \mathrm{s}^{-1}\right)\end{array}$ & $\begin{array}{c}L_{30}^{\mathrm{a}} \\
\left(\mathrm{erg} \mathrm{s}^{-1}\right)\end{array}$ \\
\hline IOC pointing non-eclipse & $0.14 \pm 0.02$ & 1.2 & $0.016 \pm 0.006$ & 0.22 \\
\hline IOC pointing primary eclipse ${ }^{c}$ & $0.046 \pm 0.019$ & 0.38 & $0.004^{\mathrm{b}}$ & $0.05^{\mathrm{b}}$ \\
\hline All-sky survey & $\begin{array}{c}0.12 \pm 0.01 \\
\text { DS Lexan/B } \\
\left(\text { count s}^{-1}\right) \quad\left(\mathrm{erg} \mathrm{s}^{-1}\right)\end{array}$ & 1.0 & $0.041^{\mathrm{b}}$ & $0.56^{\mathrm{b}}$ \\
\hline Deep Survey non-eclipse, non-flare & $0.31 \pm 0.01$ & 1.1 & - & - \\
\hline Deep Survey primary eclipse ${ }^{c}$ & $0.17 \pm 0.01$ & 0.6 & - & - \\
\hline Deep Survey secondary eclipse ${ }^{d}$ & $0.28 \pm 0.01$ & 1.0 & - & - \\
\hline
\end{tabular}

about 5 days in the telescope field of view. Objects farther from the equator were scanned for longer times, such that objects at the ecliptic poles were scanned for the full six months of the survey. AR Lac was scanned from 1992 December 16 to December 24.

Data reduction for the all-sky survey data proceeds in a way similar to that of the pointed data, although the process is complicated somewhat by the spinning attitude of the spacecraft. Unfortunately, AR Lac is sufficiently faint in the EUV to preclude detection in all but the Lexan/B filter. In this filter the count rate was too low to allow light curves to be produced. The survey Lexan/B count rate was therefore determined using the data from the entire scanning period. We list this in Table 2, together with the upper limit to the count rate derived from the $\mathrm{Al} / \mathrm{Ti} / \mathrm{C}$ survey data.

\subsection{Deep Survey Pointing}

AR Lac was observed with the EUVE Deep Survey (DS) telescope from 02:05 UT on 1993 Oct 12 until 06:08 UT on 1993 Oct 15 as part of a GO pointing by F. Walter. The observation accumulated $\sim 98$ ks once earth-blocked, daytime, and SAA intervals were excluded. The observation was centered in the Lexan/B filter. We obtained DS QPOE (quick position event) files from the $E U V E$ archive and processed the data using the most current telescope effective area, vignetting corrections and corrections for when the telemetry was busy. We obtained the DS light curve using the standard EUV IRAF software.

The first half of the pointing AR Lac was centered on a region of low gain in the detector. This feature is known locally as the "dead spot" and was caused by damage to the micro channel plates by the persistently brightest EUV source HZ 43. The position of AR Lac on the detector was moved to a nominal region of the detector for the second half of the observation, and includes the primary eclipse and a flare. The count rate for non-eclipse non-flaring emission during the second half of the observation is $0.31 \pm 0.016$ count $\mathrm{s}^{-1}$. The count rate for the persistent emission during the first half of observation is $0.15 \pm 0.01$ count $\mathrm{s}^{-1}$, which is consistent with the count rate of the second half once the correction factor of 2.1 is applied. This correction factor was calibrated fairly accurately using several different sources, including the Moon.

The flare is a factor of 2 increase over the persistent emission count rate. Count rates for the persistent emission (corrected for the dead spot), secondary and primary eclipses are given in Table 2 .

\section{RESULTS AND DISCUSSION \\ 3.1 Light Curves \\ 3.1.1 Scanner and deep survey Lexan/B}

The AR Lac Lexan/B scanner light curve is shown in Fig. 1. A Modified Julian day is plotted along the lower axis while the phase based on the ephemeris from M. Rodonò, as quoted in White et al. (1990), has been indicated at the top. (This phase agrees to within 0.002 of a phase compared to the quadratic ephemeris given by Kim (1991), less that the uncertainty in extrapolating the phase to our observing epoch.) The times of total and partial eclipse of the G star have also been indicated. The data have been binned over entire orbits to improve the signal-to-noise $(\mathrm{S} / \mathrm{N})$ ratio. A similar plot of the $\mathrm{Al} / \mathrm{Ti} / \mathrm{C}$ results is shown in Fig. 2. Because of the much smaller $\mathrm{Al} / \mathrm{Ti} / \mathrm{C}$ count rates and subsequently lower $\mathrm{S} / \mathrm{N}$, these data are not quite so useful for extracting eclipse information.

Both Lexan/B and Al/Ti/C light curves are characterized by a rise over the first two data points (each corresponding to the nighttime data from successive EUVE orbits), followed by what appear to be stochastic variations or rotational modulation over the remainder of the observation. The period covered by the data from the first EUVE orbit of the observation is entirely within the total eclipse of the G-star photosphere. The midpoint of this satellite nighttime occurs near phase 0.01 . At this phase, based on the system parameters listed in Table 1, the G star and all coronal structures less than $0.9 R_{\odot}$ above its photosphere are completely eclipsed by the K-star photosphere. At this time, the Lexan/B count rate is $0.046 \pm 0.019$ count $\mathrm{s}^{-1}$. Outside of the eclipse, the average count rate is about 0.13 count $\mathrm{s}^{-1}$.

The DS observation of AR Lac was centered in the Lex/B filter and is shown in Fig. 3. The data have been binned over each EUVE nighttime orbit for better $\mathrm{S} / \mathrm{N}$ and consistency 


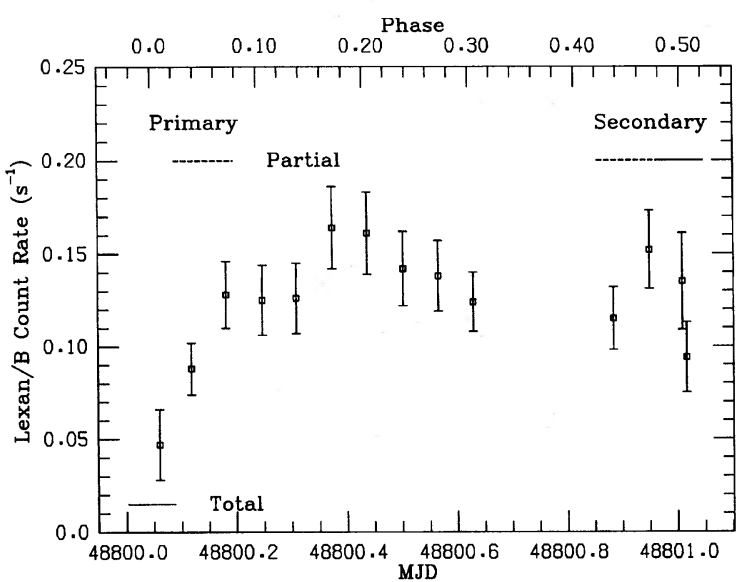

FIG, 1. AR Lac Lexan/B count rates for the pointed IOC observation. Modified Julian day is located along the bottom axis with orbital phase from Rodono, as quoted in White et al. (1990), indicated along the top. The times of total and partial eclipse of the $\mathrm{G}$ star and the secondary eclipse are indicated: the length of each bar corresponds to the duration of each particular phase, while the vertical position is arbitrary.

with the scanner analysis. The light curve covers slightly more than 1.5 AR Lac orbital periods and conveniently starts at phase 0.0. As in Fig. 1, the ephemeris from M. Rodonò [as quoted in White et al. (1990) and is indicated at the top], was found to be in good agreement with the quadratic ephemeris of Kim (1991). The first half of the observation was corrupted by the dead spot (Sec. 2.4). Primary eclipse can be seen at MJD 49274.0. The average count rate during primary eclipse is $0.25 \pm 0.005$ count $\mathrm{s}^{-1}$ with a minimum count rate of 0.17 count $\mathrm{s}^{-1}$. The secondary eclipse occurs at MJD

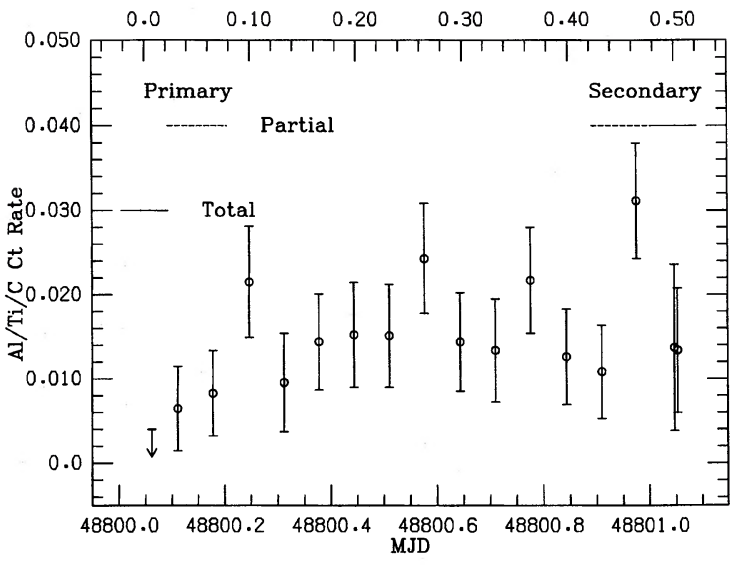

FIG. 2. Same as Fig. 1 for the Al/Ti/C filter.

49273.0 and is barely discernible from the overall variability. A flare occurs at the next expected secondary eclipse (MJD 49275.0) and is a factor of 2 increase above the persistent emission.

We note immediately that this overall pattern we see in both DS and scanner light curves is very similar to that observed using the EXOSAT low-energy imaging telescope (LEIT; White et al. 1990) the ROSAT PSPC (Ottmann et al. 1993) and ASCA (White et al. 1994) over the same range in phase. This eclipse modulation is direct evidence of EUV emission from regions with spatial dimensions less than, or comparable to, the size of the $\mathrm{K}$ star, as was first observed by Walter et al. (1983) using the Einstein IPC. Now, if the corona of the $\mathrm{G}$ star is more extended than $\sim 1 R_{\odot}$, it will not

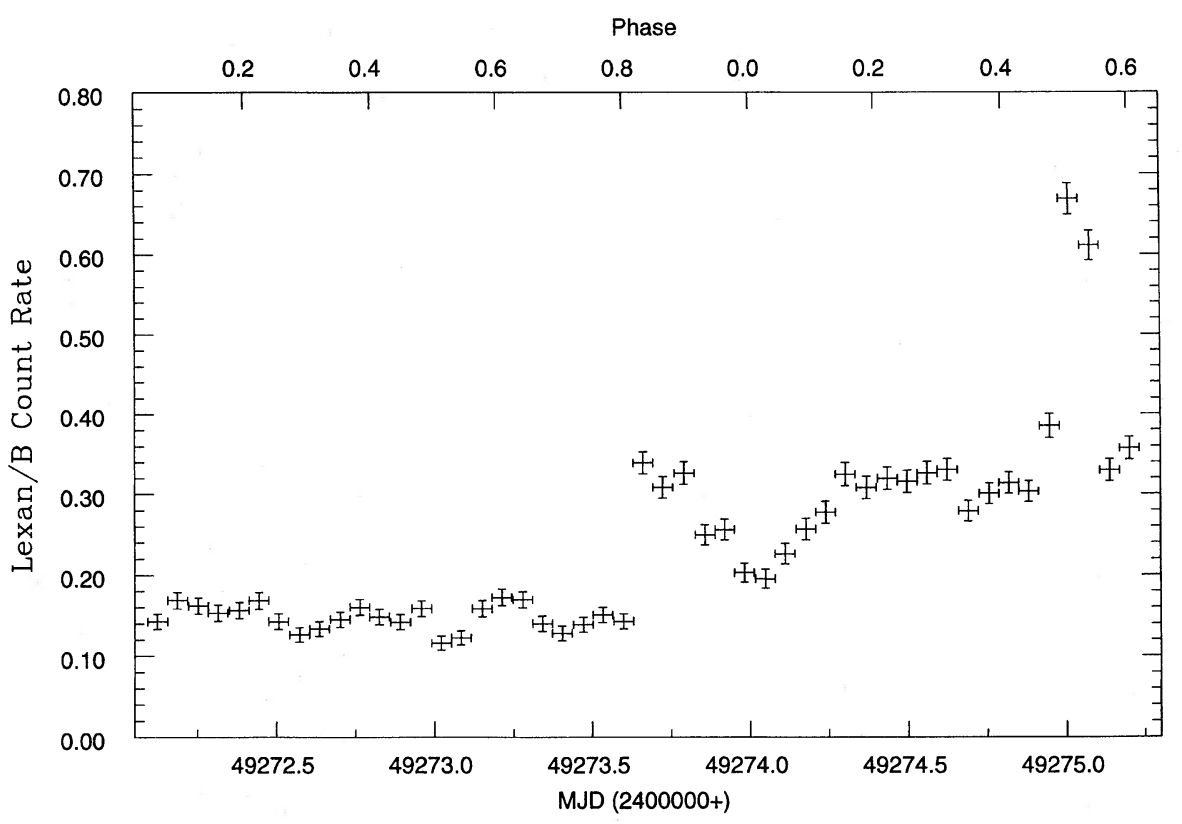

FIG. 3. Same as Fig. 1 for the Deep Survey Lexan B filter. The discontinuity in the light curve is a result of the first half of the observation having been pointed on a low gain region of the Deep Survey detector caused by the EUV bright white dwarf HZ 43 (see text). Eclipse of the K star occurs at MJD 49273.0, and is barely discernible from the overall variability. The second flare occurs during the second expected eclipse of the K star near MJD 49275.0. Eclipse of the $\mathrm{G}$ star occurs at MJD 49274.0 . 
be totally eclipsed. Therefore, based on the simplifying assumption that the coronae are stable on time scales of an orbital period and are spherically symmetrical and homogeneous, the minimum fraction of the Lexan B count rate we can attribute to the $\mathrm{G}$ star based on the scanner light curve is about two thirds, and on the DS light curve is about $\sim 40 \%$. The scanner result is superficially contrary to the findings of Walter et al. (1983), who observed a depression in the IPC count rate of only $30 \%$, similar to what we detect for the DS. They determined that the greater fraction of the x-ray emission detected originated from the $\mathrm{K}$ star. The EXOSAT LEIT light curve exhibited a decline in count rate of $\sim 50 \%$ during primary eclipse; later ROSAT and ASCA light curves indicate very similar dips (Ottmann et al. 1993; White et al. 1994). The EXOSAT LEIT observation was made with a $4000 \AA$ Lexan filter whose bandpass is similar to that of the EUVE Lexan/B filter. It is possible that, over time scales of years, the relative $G$ - and $\mathrm{K}$-star activity levels change, perhaps as a result of the behavior of the activity cycles in each star (see, Sec. 3.2 however,). In reality, the assumption of spherically symmetrical, homogeneous and constant coronae is unlikely to be entirely accurate. Both the rotational modulation of EUV-bright, compact coronal structures and the flaring activity or stochastic variability could give rise to variations similar to those we observe here in the scanner Lexan/B band. Since the temporal coverage of the IOC observation was less than one orbital cycle, and the DS observation was only slightly more than one cycle, such ambiguities cannot easily be resolved here. Evidence that the former of these effects-rotational modulation-was responsible for variation of EUV flux observed from the RS CVn system V711 Tau has recently been presented by Drake et al. (1994); however, later observations of this system showed stochastic and flaring variability but no hard evidence for rotational modulation (Drake et al. 1996a). Since outside of eclipse the EUVE IOC and survey Lexan/B count rates (Table 2) are, within the observational uncertainties, the same, we conclude that the pattern of variability we are seeing in the Lexan/B and $\mathrm{Al} / \mathrm{Ti} / \mathrm{C}$ filters is not a result of large-scale flare-like activity but likely represents the "average"' behavior of the system. In Sec. 3.2 we also show that the DS count rate outside of eclipse is consistent with the same "average", behavior. The types and combinations of stable (on orbital time scales) eclipsing and rotationally occulted coronal features that are possible are illustrated in the detailed modeling of the EXOSAT AR Lac data by White et al. (1990; see, also, Siarkowski 1992), and of the ROSAT PSPC data by Ottmann et al. (1993).

The IOC scanner observation was terminated, frustratingly, while entering the secondary eclipse. No evidence for a significant decrease in the Lexan/B count rate can be seen through the partial eclipse phase though the size of the error bars precludes a definitive judgment. While a dip in the DS light curve is seen during secondary eclipse, this is not of greater significance than the, presumably stochastic, dip which occurs nine or so hours earlier. These results provide some support for the prognosis that the majority of the Lexan/B emission arises from a compact corona on the $\mathrm{G}$ star. These data are also consistent with the diagnosis of an extended corona on the $\mathrm{K}$ star such as has been proposed in the previous studies cited above. Again, the EXOSAT LEIT observations of White et al. (1990), the ROSAT PSPC observations of Ottmann et al. (1993), and the ASCA results of White et al. (1994) are qualitatively similar to our observations and are perhaps of slightly higher quality: all the data sets show either a very shallow dip or no firm evidence for a dip at secondary eclipse, i.e., there is no strong eclipse of the $\mathrm{K}$-star corona.

Maximum entropy modeling and $\chi^{2}$ fitting of the EXOSAT data by White et al. (1990) lead, in general, to solutions with two bright regions, though the placement of these regions on either one or both stars was not constrained. One of these regions was compact, while the spatial extent of the other depended on location. The fraction of the combined total surface area of both stars covered by these regions was relatively small-less than $20 \%$ for all solutions. It would, then, seem likely that a significant evolution in the shape or location of these small, bright regions might lead to completely different light curves. It is interesting that the qualitative shape of the Lexan/B scanner light curve is very similar to that of the EXOSAT LEIT, with a maximum about 90 degrees in phase following primary eclipse. This suggests that the qualitative similarity between the EUVE and EXO$S A T$ observations, carried out 8 years apart, could be somewhat fortuitous. Alternatively, this similarity could suggest that the inhomogeneous structure in the White et al. (1990) models is stable on a time scale of years. The EUVE DS and the ROSAT PSPC light curves indicate that this is not actually the case. The ROSAT light curve (obtained in 1990) shows a much steeper egress from primary eclipse than do the EXOSAT LEIT data. The EUVE DS data show a more shallow egress. However, Ottmann et al. (1993) were still able to apply the modeling technique of White et al. (1990) to derive different configurations of coronal structures qualitatively similar to those derived by White et al. (1990) based on the EXOSAT data. Siarkowski (1992) also obtained highly structured solutions to the $3 \mathrm{D}$ modeling of the EXOSAT LEIT data. Are these structures obtained from the light curve models real? Models based on light curve data alone are highly non-unique, and one can, no doubt, construct a large variety of different plausible coronal configurations which fit the data well equally. However, the answer to this question perhaps can be found in other non-eclipsing RS CVn light curves. We note that scanner and DS light curves of the RS CVn binary V711 Tau (HR 1099) [obtained after the data published by Drake et al. (1994)] show much variability, including both flares and (apparently) stochastic non-flaring variability on time scales of hours and with amplitudes comparable to the primary eclipse dips observed in the case of AR Lac (Drake et al. 1996a). These variations do not appear to be caused by rotational modulation, unlike the variability analyzed by Drake et al. (1994). The presence of such stochastic variability indicates that detailed models of these light curves based on the assumption that the coronal structures are stable over orbital time scales overinterpret the data.

The Lexan/B scanner light curve through the primary partial eclipse phase is in reasonable agreement with a uniform, compact corona on the $\mathrm{G}$ star. However, the uncertainty in 
the Lexan/B scanner data and corruption of the DS data by the "dead spot" do not warrant further detailed modeling. The phase at the midpoint of the second EUVE orbit nighttime is about 0.03 , which is outside of totality. At this phase, a portion of the $\mathrm{G}$ star resembling a spherical cap with height $0.5 R_{\odot}$ would be visible above the K-star photosphere. If an isotropic, spherically symmetric corona of scale height $0.1 R_{\odot}$ [about twice as large as the Walter et al. (1983) model] is assumed arbitrarily, then about $20 \%$ of the stellar corona would be visible above the K-star photosphere. One $E U V E$ orbit later, most of the G-star photosphere and approximately $80 \%$ of the assumed G-star corona would be visible. The observed Lexan/B scanner count rate perhaps increases too quickly outside of eclipse for this simple model to be correct. However, it is readily imagined that a small variation on this design, including some inhomogeneities or time variability such as are most likely in the true case, could explain the Lexan/B observations with sufficient accuracy. The highly inhomogeneous models put forward by White et al. (1990), Siarkowski (1992), and Ottmann et al. (1993) with appropriate adjustments, could no doubt also be consistent with these data.

The DS Lexan/B light curve climbs rather too slowly out of primary eclipse to be explained by geometric obscuration of a homogeneous, static corona. This was pointed out by Walter (1996), who suggested that spatially extended, optically thick prominences might be responsible. However, if we again admit a degree of variability, a dip, such as those observed at orbital phases 0.3 and 0.7 around the first secondary eclipse, superimposed over the light curve at egress could also explain the data.

\subsubsection{Scanner Al/Ti/C}

Although the count rates in the scanner $\mathrm{Al} / \mathrm{Ti} / \mathrm{C}$ band are much lower than those in Lexan/B, it is still possible to discern the rise in count rate coming out of primary eclipse. During the total eclipse, there is no significant detection in the $\mathrm{Al} / \mathrm{Ti} / \mathrm{C}$ band, with the upper limit being 0.004 count $\mathrm{s}^{-1}$. There are several high points in the light curve which resemble flare-like brightenings but which are probably not statistically significant; we also should have seen sympathetic brightenings in the Lexan/B data if the high points were due to flares. Indeed, out of the eclipse phase the count rate is statistically constant with a mean of $0.016 \pm 0.006$ count $\mathrm{s}^{-1}$. In analogy to our reasoning above, we can estimate from this that at least $60 \%$ (and probably more) of the $\mathrm{Al} / \mathrm{Ti} / \mathrm{C}$ flux originates from the $\mathrm{G}$ star. Again, there is no evidence of a darkening going into the secondary eclipse. In summary, the $\mathrm{Al} / \mathrm{Ti} / \mathrm{C}$ data look the same as the scanner Lexan/B data but with lower S/N.

\subsection{Emission Measures}

Estimates of coronal temperature and emission measure on AR Lac have been made from Einstein (e.g., Swank et al. (1981); Schmitt et al. 1990), EXOSAT (e.g., Pasquini et al. 1989; White et al. 1990), ROSAT (Ottmann et al. 1993; Dempsey et al. 1993), and ASCA (White et al. 1994) data. Schmitt et al. (1990) have fitted single-temperature, two- temperature, fixed-temperature, and continuous emission measure models to the data from several Einstein IPC observations of AR Lac. Each of the models provided acceptable fits to at least one IPC observation. Typical temperatures were $6.4 \leqslant \log T \leqslant 7.4$ with emission measures $53.0 \leqslant \log E M$ $\left(\mathrm{cm}^{-3}\right) \leqslant 54.0$. In general, a two-temperature model gave the best fits to the largest number of observations. Twotemperature models were also applied to the ASCA, ROSAT, and EXOSAT data by the above mentioned authors. In all cases the two-T models produced satisfactory fits to the data.

As we have discussed above, White et al. (1990), based on the non-detection of primary eclipse in the EXOSAT ME band, argued that the low and high temperature components on AR Lac are associated with two physically distinct regions. The later ASCA and ROSAT observations do show strong primary eclipses at higher energies and are also satisfied by two-T models. However, support for the two-T models can also been found in other work (e.g., Mewe et al. 1986; Lemen et al. 1989; Pasquini et al. 1989). Nevertheless, the two-temperature fits, first proposed by Swank et al. (1981), are not entirely without controversy. Contrary to the two-T coronal model it has also been argued that the bimodal temperatures are a spurious artifact resulting from a combination of a continuous emission measure temperature distribution with the particular x-ray instrument spectral response (e.g. Majer et al. 1986; Stern et al. 1986; Montesinos \& Jordan 1993). Ottmann et al. (1993) also argued that the ROSAT PSPC data supported this view. Fortunately, we can now draw on the information from $E U V E$ spectroscopy of active stars for the solution to the two-T corona controversy. Very recently, Walter (1996) presented an EM for AR Lac based on a global plasma model fit to the EUVE spectrum. The EM distribution is continuous but does display two humps at $\log T \sim 6.7$ and 7.3. While it is tempting to associate these two humps with the two-T coronae, it is not clear from the modeling technique adopted whether or not this structure is statistically significant. In general, EUVE spectra of active and relatively inactive stars do not show evidence for two-T coronae: the EM distributions derived from EUV spectral lines are continuous (e.g., Drake 1996; Dupree et al. 1996). Nevertheless, we can use the two temperature models derived for AR Lac in earlier studies as convenient parametrizations with which to compare our EUVE photometric observations.

Unlike the proportional counters used on Einstein, EXO$S A T$, and ROSAT, the EUVE- detectors do not possess any intrinsic energy discrimination. However, the broad-band EUVE data do provide some degree of information on the emission measure as a function of temperature. For each filter we have derived "emission measure solutions." This type of approach was pioneered by Pallavicini et al. (1988) in their study of nearby coronal sources using broad band EXOSAT data and has been used in the analysis of other EUVE broad band data by Vedder et al. (1993), Patterer et al. (1993), and Drake et al. (1994; see also Vedder et al. 1994), and it is discussed in detail by Drake \& Mathioudakis (1996). In brief, by folding an optically thin radiative-loss model calculated for a range of temperatures through a given filter bandpass, we can determine the coronal emission mea- 


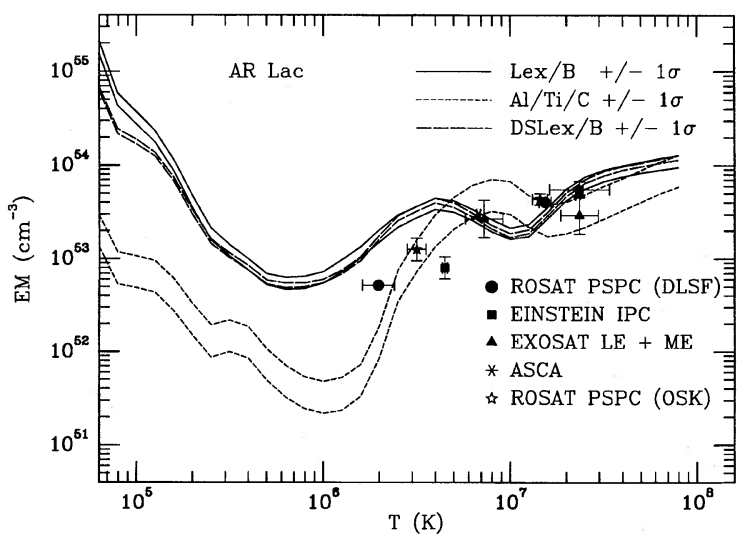

FIG. 4. Emission measure vs temperature "solutions" for AR Lac (refer to text). Also shown are the two-temperature solutions from Enstein IPC, EXOSAT LE+ME, ROSAT PSPC, and ASCA observations (see Sec. 3.2). The different sets of points corresponding to ROSAT PSPC fits are from the work of Ottman et al. (1993; OSK) and Dempsey et al. (1993; DLSF).

sure which, at any single temperature, would give rise to the observed count rate for that filter. For a given filter, then, this method yields a locus of single-temperature models in the emission measure-temperature plane. Since the locus for each filter will be different, the intersections of any two loci from different filters yield one single temperature fit. In reality the coronal plasma will not be isothermal: the emission measure-temperature loci then correspond to the emission measure upper limit as a function of temperature. In this case the interpretation of loci corresponding to different filters is not straightforward; the intersections of the loci corresponding to different filters do not, of course, represent meaningful solutions. To determine the emission measure at any given temperature, it is then necessary to know the contribution to the flux in the filter of interest from all other temperatures.

Because the count rates for the scanner IOC observation have changed from Patterer et al. (1993), as described in Sec. 2 , and the $N_{H}$ value adopted here is also significantly different, the temperature and emission measure loci have also changed. Figure 4 shows the $\pm 1 \sigma$ emission measure versus temperature loci that satisfy the corrected EUVE count rates and their uncertainties. The emission measures were calculated using the optically thin plasma radiative-loss model of Landini \& Monsignori-Fossi (1990) corresponding to solar abundances, folded through the Lexan/B and $\mathrm{Al} / \mathrm{Ti} / \mathrm{C}$ effective areas for each temperature from $\log T=4$ to $\log T$ $=8$ in 0.1 dex steps. The attenuation by the ISM was calculated using an interstellar neutral helium-to-hydrogen ratio of 0.1 and the opacities of Rumph et al. (1994). The neutral hydrogen column density was first determined using a distance of $47 \mathrm{pc}$ and a neutral hydrogen volume density of 0.07 $\mathrm{cm}^{-3}$ (Paresce 1984). However, this yields $N_{H} \sim 10^{19} \mathrm{~cm}^{-2}$, which is much too high based on Walter's (1996) detection of the Fe XVI lines at 335 and $364 \AA$. Using the ratios of these two lines, Walter estimated $N_{H} \sim 2 \times 10^{18} \mathrm{~cm}^{-2}$ and we adopt this value here. By overplotting the Lexan/B and $\mathrm{Al} /$ $\mathrm{Ti} / \mathrm{C}$ loci, acceptable single-temperature solutions appear as the regions where the $\pm 1 \sigma$ bands corresponding to the different filters overlap. These are:

$$
\begin{aligned}
& 4.0 \times 10^{6} \mathrm{~K} \leqslant T \leqslant 7.0 \times 10^{6} \mathrm{~K} \text { and } 3.0 \times 10^{53} \mathrm{~cm}^{-3} \\
& \leqslant \mathrm{EM} \sim 5.0 \times 10^{53} \mathrm{~cm}^{-3}, \\
& 1.2 \times 10^{7} \mathrm{~K} \leqslant T \leqslant 2.0 \times 10^{7} \mathrm{~K} \text { and } \mathrm{EM} \sim 3.0 \\
& \times 10^{53} \mathrm{~cm}^{-3}, \\
& T \geqslant 4 \times 10^{7} \mathrm{~K} \text { and } \mathrm{EM} \geqslant 8 \times 10^{53} \mathrm{~cm}^{-3} \text {. }
\end{aligned}
$$

We note that, while we have adopted a solar metallicity for these calculations, the indicated temperatures are insensitive to the exact coronal metallicity. If the corona of AR Lac is indeed metal deficient relative to solar, as suggested by the ASCA results of White et al. (1994) and Singh et al. (1996), then the EM loci would all be shifted upwards by an amount corresponding to the metal deficiency. This is because, throughout the $5 \leqslant \log T \leqslant 7$ temperature range, the radiative loss for an optically thin thermal plasma at EUV wavelengths is dominated by metal lines.

From these data, the two high temperatures indicated by the intersection of the scanner Lexan/B and $\mathrm{Al} / \mathrm{Ti} / \mathrm{C}$ loci cannot really be discriminated, and the two hotter components listed above should probably be considered together: $T \geqslant 1.2 \times 10^{7} \mathrm{~K}$ and $\mathrm{EM} \geqslant 2 \times 10^{53} \mathrm{~cm}^{-3}$. Note that the DS Lexan/B locus cannot be strictly compared to the scanner $\mathrm{Al} / \mathrm{Ti} / \mathrm{C}$ locus because the two observations were not simultaneous. However, the DS Lexan/B and scanner Lexan/B loci lie on top of each other, indicating that the average emission measure during the DS and scanner observations was the same. For comparison purposes, we have also plotted in Fig. 4 points corresponding to the two-temperature models derived from the Einstein and EXOSAT data, cited above, from the ROSAT PSPC all-sky survey data by Ottmann et al., (1993) and Dempsey et al. (1993), and from the ASCA data by White et al. (1994). In the cases of the Einstein IPC and EXOSAT LE+ME models, the emission measures and error bars in Fig. 4 are based on an average of models derived from three and two different observations, respectively. The ROSAT PSPC points attributed to Ottman et al. are averages of the points plotted in their Figs. 4(a) and 4(b), with uncertainties representative of the scatter of these points. Since the different authors have used different plasma models to derive these different two- $T$ models, these data points should be compared with caution, especially since the observations were all made at different times. Indeed, White et al. (1990) remark on differences of up to a factor of 3 in emission measure and 6 in temperature between models derived from their EXOSAT and the earlier Swank et al. (1981) Einstein SSS data, which might be attributable to variability. However, it is noteworthy that the agreement between the Einstein IPC, EXOSAT ME, ROSAT PSPC, and ASCA model hot components is reasonable. Also noteworthy is the agreement between these hot component emission measures and the values suggested by the EUVE loci; though, as discussed above, in the presence of a true nonisothermal corona the 
single temperature solutions lose their meaning. Nevertheless, from this comparison it is clear that the dominant $\sim 10^{7}$ $K$ emission measure in the AR Lac corona was the same within a factor of 2-3 during each of the different observations illustrated in Fig. 4.

It might be tempting to attribute the apparent lack of coronal variability over fairly long time scales to the "saturation" in stellar activity observed in the rapidly rotating stars such as the young Pleiades $\mathrm{dK}$ stars and the very active RS CVn systems (e.g., see the discussion by Sciortino 1993). The physics behind this saturation effect are not understood. Based on its x-ray flux, AR Lac is also perhaps slightly below the saturation limit for its spectral type, and we suggest that the constancy of its coronal emission is not a result of saturation, but a result of a different type of dynamo activity in the rapidly rotating AR Lac components, as compared to more slowly rotating stars like the Sun. This picture has recently been discussed by Drake et al. (1996b) and further evidence supporting this suggestion has been presented by Stern et al. (1995), whose analysis of x-ray observations of stars in the Hyades cluster indicates that they have coronae whose $\mathrm{x}$-ray emission does not vary appreciably (by more than a factor of $\sim 2$ ) over time scales of years. These Hyades stars also lie well below the observed activity saturation level. Stern et al. (1995) argue that it is a change in the nature of the magnetic dynamo in the more rapidly rotating stars which is responsible for the lack of variability through the lack of an activity cycle. The magnetic activity of the rapidly rotating stars is then generated by a turbulently driven dynamo process such as that discussed by Durney et al. (1993), Weiss (the "fibril"' dynamo; 1993) and others. In this scenario, this same dynamo process is responsible for the activity observed in the very low mass dwarfs that likely do not posess the radiative cores thought to be necessary for the solar-like large scale field $\alpha \omega$ dynamo to operate (e.g., see discussion in Drake et al. 1996b). In stars with radiative cores which have relatively high rotation rates, such as the RS CVn's, and the Hyades dwarfs discussed by Stern et al. (1995), the turbulent dynamo dominates the large scale field ("solar-like") dynamo and well-defined activity cycles which would give rise to large amplitude variability over periods of years are not observed.

\subsection{EUV Luminosities}

By assuming a spectrum model, we can derive the EUV luminosities within the Lexan/B and $\mathrm{Al} / \mathrm{Ti} / \mathrm{C}$ bandpasses. For this purpose, we have again used the plasma emissivities from Landini \& Monsignori-Fossi (1990) with solar abundances, together with the same value as before, $N_{H}=2 \times 10^{18}$ $\mathrm{cm}^{-2}$, for the intervening hydrogen column density. In this case, the use of solar abundances has very little influence on the resulting luminosities. For example, adopting a plasma metallicity of $[\mathscr{O} / H]=-0.3$ (in the usual spectroscopic notation) instead of solar $([\mathscr{C l} / H]=0)$ results in EUV luminosities which differ by only a few percent. The final luminosity is also relatively insensitive to the temperature assumed for the coronal plasma within the range $6.0<\log T<7.0$; we have used, arbitrarily, the value $\log T=7.0$ as a typical average coronal temperature indicated by the emission measure analysis described above and by Walter (1996).

The rough mean non-eclipse Scanner Lexan/B count rate of 0.12 count $\mathrm{s}^{-1}$, and the DS Lexan/B count rate of 0.31 count $\mathrm{s}^{-1}$ correspond to a luminosity of about $1 \times 10^{30}$ $\mathrm{erg} \mathrm{s}^{-1}$ in the $50-180 \AA$ band. The mean $\mathrm{Al} / \mathrm{Ti} / \mathrm{C}$ count rate of 0.016 count s ${ }^{-1}$ yields a luminosity of $0.2 \times 10^{30} \mathrm{erg} \mathrm{s}^{-1}$ in the 160-240 $\AA$ band. These luminosities are also fairly insensitive, within their observational uncertainties, to the adopted plasma emissivity model. Test calculations indicated that the Raymond \& Smith (1977) and Mewe et al. (1985) models yield luminosities which differ by less than $15 \%$ compared to those calculated using the Landini \& Monsignori-Fossi (1990) model. We have listed the final luminosities in the different bandpasses in Table 2 . Note that the DS Lexan/B and Scanner Lexan/B bandpasses are slightly different, and that the effective area of the former is larger by about a factor of 2 . Uncertainties on these values can be taken to be $\sim 20 \%$, which takes into account possible systematic errors in the plasma model adopted.

\section{CONCLUSIONS}

We have combined EUVE deep survey and scanning telescope observations taken at different times to investigate the eclipsing RS CVn system, AR Lac. In total, the observations cover approximately two orbital periods, including both primary and secondary eclipses. The deep survey observation detected a flare at the time of expected secondary eclipse. The deep survey and scanner observations show, respectively, an $\sim 40 \%$ to $\sim 60 \%$ reduction in flux, when the $\mathrm{G}$ star is eclipsed by the $\mathrm{K}$ star. This decrease is consistent with the $\mathrm{G}$ star having a compact corona, although assumptions of a spherically symmetrical, homogeneous, and constant corona are probably incorrect. There was no discernible decrease in count rate during the secondary eclipse (i.e., the $\mathrm{K}$ star eclipsed by the $G$ star), which supports the idea put forth previously that the $\mathrm{K}$ star has an extended corona. Behavior of the scanner $\mathrm{Al} / \mathrm{Ti} / \mathrm{C}$ light curve shared a pattern similar to the Lexan/B light curve. The Lexan/B count rate observed during the all-sky survey is in excellent agreement with the IOC count rate outside of the eclipse.

Our Lexan/B DS and scanner light curves are remarkably similar in shape to those derived from observations made with the EXOSAT LEIT by White et al. (1990) and with the ROSAT PSPC by Ottmann et al. (1993). The coronal modeling techniques used by these authors favored solutions corresponding to two or more bright features with relatively small surface coverage factors. The qualitative similarities between our light curves and theirs suggest that either these coronal features are grossly stable over a time scale of years or else the similarities in the light curves are purely coincidental.

An emission measure analysis using the Lexan/B and $\mathrm{Al} /$ $\mathrm{Ti} / \mathrm{C}$ count rates has yielded three single-temperature solu- 
tions. The hotter two of these have temperatures similar totwo-component models derived from Einstein, EXOSAT, and ROSAT low-resolution data. However, since the corona of AR Lac is probably not isothermal, these solutions are possibly spurious. The emission measures indicated by the $E U V E$ data are generally higher than those of the above mentioned two-component models, although again, this is possibly a result of the impropriety of the one- and twocomponent model approximations.
We are grateful for useful discussions with Brunella $\mathrm{C}$. Monsignori-Fossi and we are deeply saddened by her passing. We extend warm thanks to to John C. Raymond of the Center for Astrophysics, and to Rolf Mewe of the Netherlands Space Research Organization, for making their optically thin plasma emission codes available to us. The EUVE science team is thanked for their advice and support. This work has been supported by NASA contract No. NAS530180 .

\section{REFERENCES}

Bowyer, S., \& Malina, R. F. 1989, in Extreme Ultraviolet Astronomy, edited by R. F. Malina and S. Bowyer, p. 397

Chambliss, C. R. 1976, PASP, 88, 762

Dempsey, R. C., Linsky, J. L., Schmitt, J. H. M. M., \& Fleming, T. A. 1993 , ApJ, 413, 333

Drake, J. J. 1996 in Cool Stars, Stellar Systems and the Sun, edited by R. Pallavicini and A. K. Dupree, ASP Conf. Series (ASP, San Francisco)

Drake, J. J., et al. 1996a, in preparation

Drake, J. J., Brown, A., Patterer, R. J., Vedder, P. W., Bowyer, S., \& Guinan, E. F. 1994, ApJ, 421, L43

Drake, J. J., \& Mathioudakis, M. 1996, in preparation

Drake, J. J., Stern, R. A., Stringfellow, G., Mathioudakis, M., Laming, J. M., \& Lambert, D. L., 1996b, ApJ (in press)

Dupree, A., Brickhouse, N. S. \& Hanson, G. J. 1996 in Astrophysics in the Extreme Ultraviolet, IAU Colloquium No. 152, edited by S. Bowyer and R. F. Malina (Kluwer, Dordrecht), p. 141

Durney, B. R., De Young, D. S., \& Roxburgh, I. W. 1993, Sol. Phys., 145, 207

Hall., D. S. 1976, in Multiple Periodic Variable Stars, IAU Colloquium No. 29, edited by W. S. Fitch (Reidel, Dordrecht), p. 287

Hall., D. S., \& Henry, G. W. 1994, IAPPP Comm., 55, 51

Hall, D. S., Richardson, T. R., \& Chambliss, C. R. 1976, AJ, 81, 138

Kim, C.-H. 1991, AJ, 102, 1784

Landini, M., \& Monsignori-Fossi, B. C. 1990, A\&AS, 82, 229

Lemen, J. R., Mewe, R., Schrijver, C. J., \& Fludra, A. 1989, ApJ, 341, 474

Majer, P., Schmitt, J. H. M. M., Golub, L., Harnden, Jr., F. R., \& Rosner, R. 1986, ApJ, 300, 360

Marshall, H. 1989, in Data Analysis in Astronomy III, edited by V. di Gisu et al. (Plenum, New York), p. 169

Mewe, R., Gronenschild, E. H. B. M., \& van den Oord, G. H. J. 1985 A\&ASS, 62, 197

Mewe, R., Schrijver, C. J. Lemen, J. R., \& Bentley, R. D. 1986, Adv. Space Res., 6(8), 133

Montesinos, B., Gimenez, A., \& Fernandez-Figueroa, M. J. 1988, MNRAS 232,361

Montesinos, B., \& Jordan, C. 1993, MNRAS, 264, 900

Neff, J. E., Walter, F. M., Rodonò, M., \& Linsky, J. L. 1989, A\&A, 215, 79

Ottmann, R., Schmitt, J. H. M. M., \& Kürster, M. 1993, ApJ, 413, 710

Owen, F. N., \& Spangler, S. R. 1977, ApJ, 217, L41

Pallavicini, R., Monsignori-Fossi, B. C., Landini, M., \& Schmitt, J. H. M.

M. 1988, A\&A, 191, 109

Pasquini, L., Schmitt J. H. M. M., \& Pallavicini, R. 1989, A\&A, 226, 225
Paresce, F. 1984, AJ, 89, 1022

Patterer, R. J., Vedder, P. W., Jelinsky, P., Brown, A., \& Bowyer, S. 1993 , ApJ, 414, L57

Popper, D. M., \& Ulrich, R. K. 1977, ApJ, 217, L41

Raymond, J. C., \& Smith, B. W. 1977, ApJS, 35, 419

Rumph, T., Bowyer, S., \& Vennes, S. 1994, AJ, 107, 2108

Schmitt, J. H. M. M., Collura, A., Sciortino, S., Vaiana, G. S., Harnden, Jr., F. R., \& Rosner, R. 1990, ApJ, 365, 704

Sciortino, S. 1993, Physics of Solar and Stellar Coronae, edited by J. F. Linsky and S. Serio (Kluwer, Dordrecht), p. 211

Siarkowski, M. 1992, MNRAS, 259, 453

Singh, K. P., White, N. E., \& Drake, S. A. 1996, ApJ, 456, 766

Stern, R. A., Antiochos, S. K., \& Harnden, F. R. 1986, ApJ, 305, 417

Stern, R. A., Schmitt, J. H. M. M., \& Kahabka, P. T. 1995, ApJ, 448, 683

Strassmeier, K. G., Hall, D. G., Fekel, F. C., \& Scheck, M. 1993, A\&AS, 100,173

Swank, J. H., \& White N. E. 1980, in Cool Stars, Stellar Systems and the Sun, edited by A. Dupree (Smithsonian Astrophysical Observatory, Cambridge)

Swank, J. H., White, N. E., Holt, S. S., \& Becker, R. H. 1981, ApJ, 246, 208

Vedder, P. W., Brown, A., Drake, J. J., Patterer, R. J., Mathioudakis, M. Cully, S., \& Pettersen, B. R. 1994, in Cool Stars, Stellar Systems and the Sun, edited by J.-P. Caillault, ASP. Conf. Ser., p. 13

Vedder, P. W., Patterer, R. J., Jelinsky, P., Brown, A., \& Bowyer, S. 1993, ApJ, 414, L61

Walter, F. M. 1996 in Astrophysics in the Extreme Ultraviolet, IAU Colloquium No. 152, edited by S. Bowyer and R. F. Malina (Kluwer, Dordrecht), p. 129

Walter, F. M., Gibson, D. M., \& Basri, G. S. 1983, ApJ, 267, 665

Weiss, N. O., 1993, in Physics of Solar and Stellar Coronae, edited by J. F. Linsky and S. Serio (Kluwer, Dordrecht), p. 541

Welsh, B., Vallerga, J. V., Jelinsky, P., Vedder, P. W., Bowyer, S., \& Malina, R. F. 1989, in X-ray/EUV Optics for Astronomy and Microscopy, edited by R. B. Hoover, Proc. SPIE, 1160, 554

White, N. E., Culhane, J. L., Parmar, A. N., Kellet, B. J., Kahn, S., van den Oord, G. H. J., \& Kuijpers, J. 1986, ApJ, 301, 262

White, N. E., Culhane, J. L., Parmar, A. N., \& Sweeney, M. A. 1987, MNRAS, 227, 545

White, N. E., Shafer, R. A., Horne, K., Parmar, A. N., \& Culhane, J. L. 1990, ApJ, 350, 776

White, N. E., et al., 1994, PASJ, 46, L97 